\title{
Lung Adenocarcinoma with Lymphangitic Carcinomatosis Showing Rapid Response to Anti-PD-1 Immunotherapy: A Brief Report
}

Dothard $A^{1}$, Petty $\mathbf{W J}^{1,2,3 *}$ and Lycan $\mathrm{T}^{1,3}$

${ }^{1}$ Department of Medicine, Section on Hematology and Oncology, Wake Forest University, Winston-Salem, NC, USA

${ }^{2}$ Department of Cancer Biology, Wake Forest University, Winston-Salem, NC, USA

${ }^{3}$ Comprehensive Cancer Center of Wake Forest University, Winston-Salem, NC, USA

\begin{abstract}
Lymphangitic carcinomatosis is a difficult to treat disease entity and usually portends a poor prognosis given the advanced state in which it is often diagnosed. Treatment is usually based on standard therapy of the primary malignancy, but in most cases, is palliative in nature. This case highlights the potential for PD-1 immunotherapy as both a rapid and durable treatment for lymphangitic carcinomatosis.
\end{abstract}

Keywords: Lymphangitic carcinomatosis; No small cell lung cancer; Nivolumab

\section{Introduction}

We report the case of a 60-year-old female with lymphangitic carcinomatosis with a non-small cell lung cancer primary who initially failed crizotinib and platinum-based therapy, but whom ultimately experienced a dramatic response to nivolumab. The proximity of the cancer cells to T-cells within the lymphatics may make nivolumab and other immunotherapies well suited for treatment of this disease.

\section{Case Review}

A 60-year-old female with hypothyroidism, hypertension, fibromyalgia, and a forty-pack year smoking history presented with a five-month history of progressively worsening dyspnea, a dry cough, and bilateral infiltrates on imaging. The pulmonary infiltrates were predominantly left sided and associated with marked interlobular septal thickening which persisted despite several courses of antibiotics. During this time, several radiographic studies were read as being consistent with lymphangitic carcinomatosis. Her dyspnea worsened and she developed bilateral pleural effusions, prevascular adenopathy, and a pericardial effusion. She underwent a pericardial window and diagnostic thoracentesis with cytology revealing CK-7 and TTF-1 positivity consistent with primary lung adenocarcinoma.

Based on the malignant pericardial effusion, her staging was consistent with stage IV lung adenocarcinoma and molecular testing revealed a ROS-1 mutation but no translocation in this gene (Table 1). The patient was started on a trial of crizotinib $250 \mathrm{mg}$ twice daily, but this was discontinued after 24 days secondary to intractable nausea and vomiting. CT imaging revealed progression of lymphangitic carcinomatosis after being treated with crizotinib. The patient was then transitioned to carboplatin/pemetrexed/bevacizumab and the bevacizumab was discontinued after one cycle due to intractable nausea

\begin{tabular}{|c|c|c|}
\hline Gene & Alteration & FDA approved therapy \\
\hline Ros 1 & V2144I & Crizitonib \\
\hline KRAS & G12C & None \\
\hline TET2 & E330fs ${ }^{*}$ & None \\
\hline TP53 & G105V & None \\
\hline CTNNA1 & $\mathrm{E} 197^{*}$ & None \\
\hline HGF & $\mathrm{K} 724 \mathrm{~N}$ & None \\
\hline RBM10 & splice site $503-1 G>T$ & none \\
\hline
\end{tabular}

Table 1: Foundation one testing results for patient. and vomiting. After she received four cycles of carboplatin/pemetrexed, repeat CT imaging to assess response found worsening intralobular and interlobular thickening, increased bilateral groundglass opacities, and multiple new bilateral pulmonary nodules, consistent with progression of disease.

At the time of disease progression through carboplatin/pemetrexed, her performance status was low. She was wheelchair-dependent due to severe dyspnea on minimal exertion despite home oxygen at $6 \mathrm{~L}$ by nasal cannula. She was incapable of self-care and options discussed in clinic included hospice referral or attempting additional treatment. After discussing risks and benefits, she opted to try nivolumab $3 \mathrm{mg} /$ $\mathrm{kg}$ IV every two weeks. The patient began to immediately note clinical improvement with improved dyspnea on follow-up clinic visit two weeks after initiating therapy. At her second follow-up visit, she was no longer wearing supplemental oxygen. After the fourth cycle of nivolumab, repeat CT imaging showed dramatic improvement in the interlobar thickening and ground glass opacities as well as disappearance of previously noted pulmonary nodules as shown in Figure 1. The patient was continued on maintenance treatment with nivolumab $3 \mathrm{mg} / \mathrm{kg}$ every 2 weeks. Ongoing CT imaging has shown continued improvement with almost complete resolution of her interlobar thickening (Figures 1A-1C). At the current time, the patient has been receiving nivolumab for over two years with near-complete radiographic response.

\section{Discussion}

Lymphangitic carcinomatosis is the permeation of malignant cells into the lymphatics to the point of obstruction, most commonly occurring within the chest. While $30 \%$ to $40 \%$ of malignancies will involve intrathoracic spread, only $6 \%$ to $8 \%$ of these cases comprise lymphangitic carcinomatosis [1]. The most common primary

*Corresponding author: W Jeffrey Petty, M.D., Wake Forest School of Medicine Medical Center Boulevard, Winston-Salem, NC 27157, USA, Tel: 336 713-4392; Fax: 336 716-5687; E-mail: wpetty@wakehealth.edu

Received October 26, 2017; Accepted November 06, 2017; Published Novembe 08, 2017

Citation: Dothard A, Lycan T, Petty WJ (2018) Lung Adenocarcinoma with Lymphangitic Carcinomatosis Showing Rapid Response to Anti-PD-1 Immunotherapy: A Brief Report. J Mol Biomark Diagn 9: 370. doi: 10.4172/21559929.1000370

Copyright: ( 2018 Dothard A, et al. This is an open-access article distributed under the terms of the Creative Commons Attribution License, which permits unrestricted use, distribution, and reproduction in any medium, provided the original author and source are credited. 

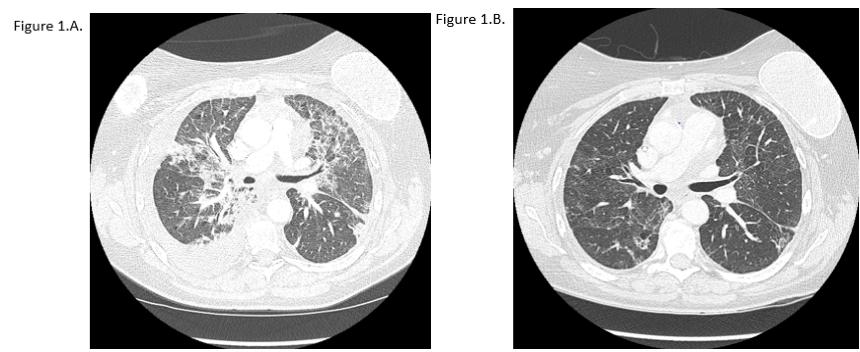

Figure 1.c.

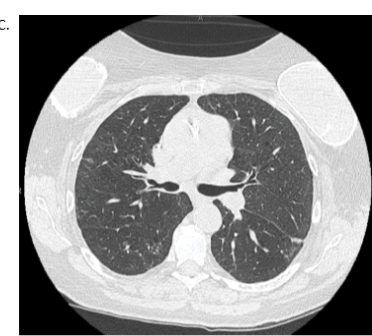

A: Initial CT chest before treatment with nivolumab showing diffuse interlobar and intralobar thickening with scattered roundglass opacities, central bronchial thickening, and right pleural effusion.

B: Repeat imaging after nearly two months of treatment with nivolumab. C: Repeat imaging after 20 months of treatment.

Figure 1: Contrast-enhanced computed tomography of the chest- lung window.

tumors that cause lymphangitic carcinomatosis are breast (33\%), stomach $(29 \%)$, lung (17\%), pancreas $(4 \%)$, prostate $(3 \%)$, cervix (3\%) and large bowel (2\%) [2]. Recent case reports have also shown lymphangitic carcinomatosis secondary to renal, liver, lip, and other head and neck cancers [3-7]. A majority are histologically comprised of adenocarcinomas [2]. Clinically, the non-specific presentation nearly always includes dyspnea with or without cough; it can also include shallow tachypnea, muscle wasting, cyanosis, and pulmonary hypertension [2]. Pulmonary functioning testing may reveal a restrictive lung pattern and diffusion deficit $[2,7]$. The prognosis of pulmonary lymphangitic carcinomatosis is particularly grim with approximately $50 \%$ to $85 \%$ of patients dying within 3-6 months of diagnosis [1,7].

Our patient presented with lymphangitic carcinomatosis due to stage IV ROS-1 mutant adenocarcinoma of the lung that was initially unable to tolerate crizotinib and whose disease had failed platinumbased therapy. At the time of the patient's presentation, docetaxel had been largely considered standard second-line therapy for those with advanced nonsmall cell lung cancer (NSCLC) failing platinum-based therapy. However, Brahmer et al. had just published their phase III study comparing the novel monoclonal antibody nivolumab to docetaxel for second-line treatment of advanced squamous NSCLC. This study found that nivolumab was superior in terms of overall survival (9.2 vs. 6 months, HR $0.59,95 \%$ CI $0.44-0.79, \mathrm{P}<0.001)$, response rate ( $20 \%$ vs. $9 \%, \mathrm{P}=0.008$ ), and progression-free survival (3.5 vs. 2.8 months; HR $0.62,95 \%$ CI $0.47-0.81, \mathrm{P}<0.001)[8]$. Following the start of therapy and dramatic response of our patient, another phase III study comparing nivolumab to docetaxel for second-line treatment of advanced nonsquamous NSCLC. This study also found that nivolumab was superior in terms of overall survival (12.2 vs. 9.4 months; HR, 0.73, 95\% CI $0.59-0.89, \mathrm{P}=0.002)$ and response rate $(19 \%$ vs. $12 \%, \mathrm{P}=0.02)$. However, median progression-free survival was greater in docetaxel than nivolumab (4.2 vs. 2.3 months), although the rate of progression free survival at one year was greater with nivolumab (19\% vs. $8 \%$ ) [9]. While this second phase III study confirmed benefit in patients with nonsquamous NSCLC, the differences in progression free survival between the two studies was incongruent. It is thought that this may partly be attributed to the increased efficacy of nivolumab with increasing PD-1 ligand expression seen in those with non-squamous NSCLC and not squamous NSCLC and may ultimately be indicative of the mutational burden difference between the two groups [10].

As previously stated, the prognosis of lymphangitic carcinomatosis remains dismal owing to the advanced stage of malignancy that is a usual part of its presentation. Often associated with poor performance status, patients with lymphangitic carcinomatosis are often considered for best supportive care alone to palliate symptoms as much as possible. However, there are reports of response to treatment with chemotherapy. Kikushi et al. documented a case of survival of 14 months after treatment with cisplatin and TS- 1 in a case of lymphangitic carcinomatosis with unknown primary [11]. Another report showed a positive response to high-dose etopiside and cisplatin in lymphangitic carcinomatosis with lung adenocarcinoma primary [12]. Fujiwara et al. showed an almost complete response that was sustained for 7.8 months with the EGFRTK inhibitor geftinib in a patient with advanced lung adenocarcioma and associated lymphangitic carcinomatosis [13]. Clinically, the treatment of lymphangitic carcinomatosis is usually predicated upon standard treatment of the primary malignancy. However, to the best of our knowledge this is the first documented response of lymphangitic carcinomatosis to immunotherapy.

Nivolumab is a monoclonal IgG4 antibody that blocks programmed cell death protein 1 (PD-1) on the surface of activated T-cells. By blocking the PD-1 immune checkpoint, the T-cells remain activated and can attack tumor cells at a higher rate whenever they are in close proximity. Since they rely on these cell-cell interactions for their mechanism, checkpoint inhibitors such as nivolumab tend to have a slower time to response than traditional chemotherapies which rapidly perfuse the tumor with cytotoxic chemicals. However increased interaction of T-cells and tumors cells may increase the speed and efficacy of checkpoint inhibitors. Several studies are examining the role of tumor-infiltrating lymphocytes in potentiating the effects of nivolumab due to this close proximity. Conversely, lymphangitic carcinomatosis is characterized by lymph-infiltrating cancer cells which theoretically should also potentiate the effects of nivolumab. When a patient presents with acute illness due to lymphangitic carcinomatosis, the time to response must be considered. Based on our experience her, rapid responses in this uncommon variant of NSCLC may be achievable with anti-PD-1 immunotherapy.

\section{Conclusion}

In the setting of patients with symptomatic lymphangitic carcinomatosis, a rapid clincal response is needed. Responses to nivolumbab often require months to manifest on imaging. However, our experience indicates that some patients with lymphangitic carcinomatosis may achieve a clinical response to nivolumab that is both rapid and durable. The biological basis for this warrant further investigation, but our case illustrates that nivolumab may provide a valuable option for the treatment of lymphangitic carcinomatosis with lung primary.

\section{Conflict of Interest and Funding}

No conflicts of interest and funding were declared by the authors.

\section{References}

1. Bruce DM, Heys SD, Eremin O (1996) Lymphangitis carcinomatosa: A literature review. J R Coll Surg Edinb 41: 7-13.

2. Yang SP, Lin CC (1972) Lymphangitic carcinomatosis of the lungs. The clinica significance of its roentgenologic classification. Chest 62: 179-187. 
Citation: Dothard A, Lycan T, Petty WJ (2018) Lung Adenocarcinoma with Lymphangitic Carcinomatosis Showing Rapid Response to Anti-PD-1 Immunotherapy: A Brief Report. J Mol Biomark Diagn 9: 370. doi: 10.4172/2155-9929.1000370

3. Babu S, Satheeshan B, Geetha M, Salih S (2011) A rare presentation of pulmonary lymphangitic carcinomatosis in cancer of lip: Case report. World $J$ Surg Oncol 9: 77.

4. Guddati AK, Marak CP (2012) Pulmonary Lymphangitic Carcinomatosis due to renal cell carcinoma. Case Rep Oncol 5: 246-252.

5. Tighe D, Cavilla S, Simcock R (2014) Pulmonary lymphangitic carcinomatosis from head and neck squamous cell carcinoma. Int J Oral Maxillofac Surg 43: 806-810

6. Wallach JB, McGarry T, Torres J (2011) Lymphangitic metastasis of recurrent renal cell carcinoma to the contralateral lung causing lymphangitic carcinomatosis and respiratory symptoms. Curr Oncol 18: 35-37.

7. Zhuang L, Liu X, Hu C, Zhang L, Jiang G, et al. (2014) Pulmonary lymphangitic carcinomatosis in liver carcinoma: a rare case report and literature review. World J Surg Oncol 12: 66.

8. Brahmer J, Reckamp KL, Baas P, Crinò L, Eberhardt WE, et al. (2015)
Nivolumab versus docetaxel in advanced squamous-cell non-small-cell lung cancer. N Engl J Med 373: 123-135.

9. Borghaei H, Paz-Ares L, Horn L, Spigel DR, Steins M, et al. (2015) Nivolumab versus docetaxel in advanced nonsquamous non-small-cell lung cancer. N Engl J Med 373: 1627-1639.

10. Ellis $P$, Vella $E$, Ung $Y(2017)$ Immune checkpoint inhibitors for patients with advanced non-small cell lung cancer: A systemic review. Clinical Lung Cancer 18: 444-459.

11. Kikuchi N, Shiozawa T, Ishii $Y$, Satoh $H$, Noguchi M, et al. (2007) A patient with pulmonary lymphangitic carcinomatosis successfully treated with TS-1 and cisplatin. Intern Med 46: 491-494.

12. Fujita J, Yamagishi Y, Kubo A, Takigava K, Yamaji Y, et al. (1993) Respiratory failure due to pulmonary lymphangitis carcinomatosis. Chest 103: 967-968.

13. Fujiwara K, Kiura K, Ueoka H, Tabata M, Hamasaki S, et al. (2003) Dramatic effect of ZD1839 ('Iressa') in a patient with advanced non-small-cell lung cancer and poor performance status. Lung Cancer 40: 73-76. 